\title{
Correction to: System inference for the spatio-temporal evolution of infectious diseases: Michigan in the time of COVID-19
}

\author{
Z. Wang ${ }^{1} \cdot$ X. Zhang ${ }^{1} \cdot$ G. H. Teichert ${ }^{1} \cdot$ M. Carrasco-Teja ${ }^{1} \cdot$ K. Garikipati ${ }^{1}$
}

Published online: 30 September 2020

๑) Springer-Verlag GmbH Germany, part of Springer Nature 2020

\section{Correction to: Comput Mech https://doi.org/10.1007/s00466-020-01894-2}

The original article was published with errors in some sentences. The correct sentences are provided in this correction.

The word "rate" in the sentence "It follows that $\beta(t) / \mu(t)$ is the effective reproduction rate: the total number..." under the section " 2 The compartmental model of infectious disease dynamics" should read as "number".

The sentence "The F-test is achieved through the following algorithm" below the equation 20 should read as "The F-test is achieved through the application of Algorithm 2".

Also, under the section "6.1 Systemidentification and ODE-constrained Optimization", the word "rate" in the sentence "...rapid decline of the effective reproduction rate, $r 0(t) . "$ in the third paragraph should read as number". The word "East" in the sentence "By contrast, Washtenaw County, about $50 \mathrm{~km}$ to the East, but" in the fourth paragraph should read as "west".

Under the section "6.2 Deep and Bayesian neural networks" the word "Appendix" in the sentence "Regions are given in Appendix..." in fourth paragraph should read as "Appendices"

In the fifth paragraph, the word "rate" in the sentence "The effective reproduction rate $r 0(t) \ldots$... should read as "number" and the word "Appendix" in the sentence "The regional results in Appendix "DNN" should read as "Appendices".

The word "rate" in the caption of Fig. 6 "Parameters of time-dependent SIRD coefficients, $\beta(t), \mu(t), \alpha(t)$, and the

The original article can be found online at https://doi.org/10.1007/ s00466-020-01894-2.

\section{K. Garikipati}

krishna@umich.edu

1 Mechanical Engineering, Mathematics and the Michigan Institute for Computational, Discovery and Engineering, University of Michigan, Ann Arbor, USA effective reproduction rate, $r 0(t)$, for Regions $1-8$ (see Fig. 1) of Michigan" should read as "number".

In the seventh paragraph, the word "rate" in the sentence "...effective reproduction rate $r 0(t)$ " should read as "number".

The Eqs. 43, 44 and 45 was published incorrectly. The correct equations are provided below.

$$
\begin{aligned}
& \mathcal{D}_{\mathrm{s}}(\mathrm{t})=\theta 16+\theta 17 \mathrm{t}+\theta 18 \mathrm{t} 2+\theta 19 \mathrm{t} 3 \\
& \mathcal{D}_{\mathrm{i}}(\mathrm{t})=\theta 20+\theta 21 \mathrm{t}+\theta 22 \mathrm{t} 2+\theta 23 \mathrm{t} 3 \\
& \mathcal{D}_{\mathrm{r}}(\mathrm{t})=\theta 24+\theta 25 \mathrm{t}+\theta 26 \mathrm{t} 2+\theta 27 \mathrm{t} 3
\end{aligned}
$$

The word "rate" under the section "7.3 Results of system identification of two dimensional SIRDmodel with diffusion" in the fourth line should read as "number" and the sentence "While the preliminary nature of these warrants caution..." should read as "While the preliminary nature of these results warrants caution..".

The word "open" in the caption of Fig. 16 "Regions 1-8: Time-dependent coefficients identified by DNNs, where an increased infection rate after the open $(\mathrm{O})$ of lockdown on June 1st is observed" should read as "opening".

The word "open" in the caption of Fig. 18 "Regions 18: Time-dependent coefficients identified by BNNs, where an increased infection rate after the open $(\mathrm{O})$ of lockdown on June 1 st is observed. Bands correspond to \pm standard deviation over the mean" should read as "opening".

Original article has been corrected.

Publisher's Note Springer Nature remains neutral with regard to jurisdictional claims in published maps and institutional affiliations. 